Chronic Obstructive Pulmonary Diseases: Journal of the COPD Foundation

\title{
Editorial
}

\section{Hospital Readmissions for COPD: We Can Meet the Challenge}

\author{
Sidney S. Braman, MD, FCCP ${ }^{1}$
}

\begin{abstract}
Abbreviations: U.S. Centers for Medicare and Medicaid Services, CMS; diagnostic-related groups, DRG; U.S Patient Protection and Affordable Care Act, ACA; Hospital Readmissions Reduction Program, HRRP

Funding Support: Not applicable

Citation: Braman SS. Hospital readmissions for COPD: We can meet the challenge. J COPD F. 2015; 2(1): 4-7. doi: http://doi. dx.org/10.15326/jcopdf.2.1.2015.0130
\end{abstract}

1 Icahn School of Medicine at Mount Sinai, New York, New
York

Address correspondence to:

Sidney S. Braman, MD, FCCP

Sidney.braman@mssm.edu

\section{Keywords:}

COPD; hospital readmissions

\section{Introduction}

Starting in October 2014, hospitals across the country are being judged on their ability to reduce 30-day, allcause unplanned readmissions for COPD after an initial admission for a COPD exacerbation. The U.S. Centers for Medicare and Medicaid Services (CMS) will penalize hospitals for what they consider excessive admissions. CMS will calculate the penalties using 3 years of hospital discharge data for COPD readmissions and compare it to the national average after adjustment for patient case-mix. ${ }^{1}$ Aggregate payments for excessive COPD readmissions as a percentage of diagnostic-related groups (DRG) payments for all discharges will be used for the calculation. In 2015, the maximum reduction possible of a hospital's aggregate Medicare payments is $3 \%$. The objectives of this initiative are laudable: reduce costs, eliminate waste, and encourage standardization of inpatient care with evidence-based protocols. By holding doctors and hospitals responsible for cost efficiency as well as high quality care, it is anticipated that patient-centered, value-driven care can be achieved.

Hospitals were given ample lead time to gear up for the penalties for COPD readmissions. In 2010, the U.S Patient Protection and Affordable Care Act (ACA) identified preventable hospital admissions as a target for improved care and cost savings. CMS launched the Hospital Readmissions Reduction Program (HRRP) in 2012, initially targeting heart failure, pneumonia and acute myocardial infarction. COPD was added to this list of conditions in 2013, a time when 1 in 5 patients were readmitted within 30 days following an index admission for a COPD exacerbation. ${ }^{2}$ Despite the potential benefits that are anticipated from this program, a number of concerns were raised before the start date of the penalty period. ${ }^{3}$ The CMS ruling assumes that high quality care that includes evidencebased treatment protocols, patient and family education programs, and a coordinated transition from hospital to community can reduce readmission rates. Yet for COPD patients, there is no clear link between quality of care and 30-day hospital readmission rates and no widely studied interventions for COPD exacerbations that have significantly reduced readmission rates. ${ }^{4}$ In addition, there is no assurance that reducing readmission rates will benefit and not harm overall patient heath status and outcomes. As a result, doubt has been expressed concerning the validity of a 30-day readmission rate as a measure of hospital quality of care and whether, given the absence of proven evidenced-based interventions, readmission rates can be reduced. ${ }^{3-6}$

Furthermore, it has been determined that COPD exacerbations disproportionately affect minorities and individuals of lower socioeconomic status. ${ }^{7}$ Hospitals that care for these patient demographic groups have 
higher readmission rates and are more likely to be penalized by CMS. $^{8}$ This includes inner-city hospitals and major teaching hospitals that share a larger burden of care for underserved populations. Factors associated with low socioeconomic status such as Medicaid insurance, low income, unstable home environment, a rural address, lack of social support, being unmarried, risk behaviors including smoking and cocaine use, poor health literacy, poor nutrition, and non-adherence to medications and follow-up visits have all been identified to predict high readmission rates. 9,10 This might lead to large financial losses and unexpectedly limit resources that would otherwise be supporting indigent care. To date CMS has not included socioeconomic factors in the risk adjustment of hospital performance measures and there is no indication that this will ever occur.

Despite the limitations that challenge hospitals and physicians to meet CMS standards on COPD readmissions, and the reservations expressed by the medical community, the penalty period has started. In response to this challenge, the COPD Foundation, whose mission is "to speed innovations that will make treatments more effective and affordable and undertake initiatives that result in expanded services for COPD patients," convened a multi-stakeholder National COPD Readmissions Summit in October 2013. Recommendations from this Summit are reported in this issue of the Journal. The Foundation is to be applauded, not only for initiating this effort, but also for the inclusive list of stakeholders. The group not only included clinicians and health service researchers but also patients, representatives of industry, and payers. The Readmissions Summit group acknowledged the paucity of published evidence to guide clinicians and hospital staff in avoiding hospital readmissions following COPD exacerbations.

Despite the lack of evidence-based measures that ensure improvement in patient outcomes and prevention of a 30-day COPD readmission, the 2013 National COPD Readmissions Summit participants provided a group of practical suggestions for best hospital practices. This included a recommendation for a coordinated transitional care, and for patientcentered efforts that include self-management skills specific to COPD. They stressed that efforts need to be made to promote patient safety and avoid adverse events. Their recommendations also remind clinicians that comorbidities are common in COPD patients and may contribute to a readmission within the 30-day period. In fact, the majority of COPD readmissions have been caused by conditions other than a reexacerbation of COPD. ${ }^{2}$ Conditions such as pneumonia, congestive heart failure, acute myocardial infarction, acute pulmonary edema, arrhythmia, gastrointestinal bleed, and metabolic problems are some of the causes of readmission and preemptive attention to these conditions during the index admission is important.

Unfortunately, many of the suggestions offered by the Summit committee have been tried and have not been shown to improve COPD readmission rates. For example, a single center, randomized trial conducted at Henry Ford Hospital was published after the COPD Readmissions Summit. ${ }^{11}$ Patients were randomized either to the control (standard care) or a bundle group in which patients received several measures: smoking cessation counseling, screening for gastroesophageal reflux disease and depression or anxiety, standardized inhaler teaching, and a 48-hour post-discharge phone call. The risk of Emergency Department visits or hospitalizations within 30 days was not different between the groups.

The COPD Foundation is developing a learning collaboratory to share best practices and to develop multicenter studies to determine what measures can prevent COPD readmissions. What can hospitals and practitioners do in the meantime? A number of institutions around the country have successfully reduced COPD readmissions. Unpublished experiences from 2 groups can be reviewed in the COPD Readmissions Summit report in this issue of the Journal. Until further research offers a standard protocol that we can use with confidence of success, attention to 4 crucial elements of transitional care can be useful: (1) Use of COPD guideline-directed treatment protocols for Emergency Department visits and hospitalized patients, ${ }^{12-14}$ (2) patient/caregiver education on smoking cessation, inhaler use and action plans for an exacerbation, (3) patient assessment of oxygen needs, comorbidities, goals of care and spirometry testing, and (4) a Follow-up Plan that includes a provider visit within 7-10 days, a post discharge phone call at 4872 hours, pulmonary rehabilitation when available, and appropriate use of community home care services. Clinicians may want to consider outpatient testing for many of the comorbidities associated with COPD such as sleep apnea, osteoporosis and coronary artery disease. Should a patient see a pulmonologist in this post-hospital period? In a study from Israel, the impact 
of a pulmonologist follow-up visit during the month after discharge from the hospital due to COPD exacerbation has recently been reported. Patients who did not see a pulmonologist had a significant increased risk for rehospitalization within 90 days from discharge. ${ }^{15}$ Whether this practice would have impact in the United States is not known at this time.

A central goal of the Obama Administration's ACA is to establish a clear timeline for moving from volume to value in the Medicare system. This has been a major transformation for hospitals, health facilities and physicians. The Hospital Readmissions Reduction Program is just one example of how the practice of Medicine must change to meet these goals. While we do not have a proven formula to reduce COPD readmissions, many of the elements, individually and collectively, have solid clinical backing that allows us to meet the challenge. 


\section{References}

1. Hospital Readmission Reduction Program. Centers for Medicare and Medicaid Web site. http://www.cms.gov/Medicare/ Medicare-Fee-for-Service-Payment/AcuteInpatientPPS/ Readmissions-Reduction-Program.html. Accessed January 25, 2015.

2. Jencks SF, Williams MV, Coleman EA. Rehospitalizations among patients in the medicarefFee-for-service program. New Eng J Med. 2009;360:1418-1428. doi: http://dx.doi.org/10.1056/NEJMsa0803563.

3. Feemster LC, Au DH. Penalizing hospitals for chronic obstructive pulmonary disease readmissions. Am J Resp Crit Care Med. 2014; 189(6): 634-639. doi: http://dx.doi.org/10.1164/rccm.201308-1541PP.

4. Prieto-Centurion V, Markos MA, Ramey NI, et al. Interventions to reduce rehospitalizations after chronic obstructive pulmonary disease. exacerbations: a systematic review. Ann Am Thorac Soc. 2014;11(3):417-424. doi: http://dx.doi.org/10.1513/AnnalsATS.201308-254OC .

5. Axon RN, Williams MV. Hospital readmission as an accountability measure. JAMA. 2011;305(5):504-505. doi: http://dx.doi.org/doi:10.1001/jama.2011.72 .

6. Joynt KE, Jha AK. Thirty-day readmissions-truth and consequences. N Engl J Med. 2012;366:1366-1369. doi: http://dx.doi.org/10.1056/NEJMp1201598 .

7. Eisner MD, Blanc PD, Omachi TA, et al. Socioeconomic status, race and COPD health outcomes. J Epidemiol Community Health. 2011;65(1):26-34. doi: http://dx.doi.org/10.1136/jech.2009.089722 .

8. Sjoding MW, Cooke CR. Readmission penalties for chronic obstructive pulmonary disease will further stress hospitals caring for vulnerable patient populations. Am J Respir Crit Care Med. 2014;190(9):1072-1074. doi: http://dx.doi.org/10.1164/rccm.201407-1345LE

9. Calvillo-King L, Arnold D, Eubank KJ, et al. Impact of social factors on risk of readmission or mortality in pneumonia and heart failure: systematic review. $J$ Gen Intern Med. 2013;28(2):269-282.

doi: http://dx.doi.org/10.1007/s11606-012-2235-x

10. Prieto-Centurion V, Gussin HA, Rolle AJ, Krishnan JA. Chronic obstructive pulmonary disease readmissions at minority-serving institutions. Ann Am Thorac Soc. 2013;10 (6):680-684. doi: http://dx.doi.org/10.1513/AnnalsATS.201307-223OT .

11. Jennings JH, Thavarajah K, Mendez M, Eichenhorn M, Kvale P, Yessayan L. Pre-discharge bundle for patients with acute exacerbations of chronic obstructive pulmonary disease to reduce readmissions and emergency department visits: A randomized, controlled trial. Chest. 2014 Dec 24. [published online ahead of print]. doi: http://dx.doi.org/10.1378/chest.14-1123 .

12. Rowe BH, Bhutani M, Stickland MK, Cydulka R. Assessment and management of chronic obstructive pulmonary disease in the emergency department and beyond. Expert Rev Respir Med. 2011;5(4):549-559. doi: http://dx.doi.org/10.1586/ers.11.43 .
13. Global strategy for the diagnosis, management, and prevention of chronic obstructive pulmonary disease (Updated 2015). Global Initiative for Chronic Obstructive Lung Disease (GOLD) Web site. www.goldcopd.com. Accessed January 29, 2015.

14. Celli BR, MacNee W, and committee members. Standards for the diagnosis and treatment of patients with COPD: a summary of the ATS/ERS position paper. Eur Respir J. 2004; 23(6): 932-946. doi: http://dx.doi.org/10.1183/09031936.04.00014304

15. Gavish R, Levy A, Dekel OK, MD, et al. The association between hospital readmission and pulmonologist follow-up visits in patients with chronic obstructive pulmonary disease. Chest. 2015 Jan 22. [published online ahead of print]. doi: http://dx.doi. org/ doi:10.1378/chest.14-1453 